m29.p03

\section{Monte-Carlo simulation of the incommensurate structure of p-Azoxyphenetol}

\author{
Eric Germaneau, Gervais Chapuis \\ École Polytechnique Fédérale de Lausanne (EPFL), Switzerland
}

\section{Keywords: disordered materials, incommensurate crystals, molecular dynamics, computational methods}

The aim of this work is to describe the behavior of an incommensurate molecular crystal using molecular dynamics simulations (MD). Incommensurate crystals are part of the more general class of aperiodic material. In addition to diffraction methods, molecular dynamical methods can be applied in order to give some information on the mechanisms leading to the aperiodicity of crystalline structures. Incommensurate crystals exhibit sometimes characteristics of disorder which should also be included in the modelling. The calculations are performed with the parallel code $d d g m q$ [1]. The compound is p-Azoxyphenetol (PAP) [2]. This compound exhibits two distinct crystalline phases from melting down to $100 \mathrm{~K}$. Phase II, stable above $356 \mathrm{~K}$ is described by a triclinic space group. Phase $\mathrm{I}$ is incommensurately modulated. The disorder is due to the distribution of the oxygen of the azoxy group on two possible sites. Currently a model has been investigated in order to determine the correct sequence of the oxygen position with the development of a code based on the metropolis algorithm. Our aim is to find the configuration with the lowest energy. This structure will be used to initiate the molecular dynamic simulations.

[1] D. Brown, H. Minoux and B. Maigret, Comp. Phys. Comm. (1997), 103, 170-186.

[2] C.B. Pinheiro, M. Gardon, P. Pattison and G. Chapuis, Ferroelectrics (2004). 305, 83-87. m29.p04

\section{Recent Advances in Crystal Structure Prediction: The Prediction of Enantiopure Separation}

\author{
Matthew D. Gourlay, John Kendrick, Frank J.J. Leusen
}

Institute of Pharmaceutical Innovation, University of Bradford, Bradford BD7 1DP, United Kingdom.Email: m.d.gourlay@bradford.ac.uk Tel: +44(0)1274236105 Fax: +44(0)1274236166

\section{Keywords: computational crystallography, energy minimization, enantioselective crystallization}

Most pharmaceutically active compounds are chiral and the different enantiomers have different physiochemical interactions in biological organisms. After the full consequences of this were realised the pharmaceutical industry has been investigating ways to purify and separate its chiral products. This has generated a lot of research into asymmetric synthesis, chiral HPLC and crystallisation. Crystallisation is considered the most economical and most efficient method of separation. A small percentage of compounds spontaneously form enantiopure crystals of the $\mathrm{R}$ and $\mathrm{S}$ enantiomers, with these systems it is possible by seeding to grow crystals of pure $\mathrm{R}$ or $\mathrm{S}$. This is a powerful method of enantiomer separation. However, the physical processes are not fully understood. In this presentation we investigate 4, and 5-hydroxymethyl-2-oxazolidinone (HMO), of which the 5HMO is used as a precursor in the manufacture of Linezolid, an antibacterial. 5HMO has been found to spontaneously form enantiopure crystals without the use of additives, whereas 4HMO forms racemic crystals.[1] These two molecules differ in structure solely by the position of the hydroxyl methyl group. We have used crystal structure prediction methods to investigate these properties, using molecular mechanical lattice minimisation to predict the lattice energies of the crystals. In both examples we predict the racemic crystal to have the lowest lattice energy. In the case of $4 \mathrm{HMO}$ this corresponds to one of the experimentally determined crystals. The lowest energy enantiopure crystal in 4HMO corresponds to another experimentally determined structure, however for $5 \mathrm{HMO}$ the experimental structure is ranked higher in energy.

[1] Pallavicini, M.; Bolchi, C.; Di Pumpo, R.; Fumagalli, L.; Moroni, B.; Valoti, E.; Demartin, F. Tetrahedron: Asymmetry 2004, 15, $1659-1665$. 